DOI: $10.5846 /$ stxb201211271684

李强, 周道玮, 陈笑莹. 地上枯落物的累积、分解及其在陆地生态系统中的作用.生态学报, 2014,34(14) :3807-3819.

Li Q, Zhou D W , Chen X Y.The accumulation, decomposition and ecological effects of above-ground litter in terrestrial ecosystem. Acta Ecologica Sinica, 2014,34(14):3807-3819.

\title{
地上枯落物的累积、分解及其在陆地 生态系统中的作用
}

\author{
李 强 ${ }^{1,2}$, 周道玮 ${ }^{1, *}$ ，陈笑莹 ${ }^{1,2}$ \\ (1. 中国科学院东北地理与农业生态研究所,长春 $130102 ; 2$. 中国科学院大学,北京 100049)
}

\begin{abstract}
摘要: 了解陆地生态系统地上枯落物的累积和分解过程对认识它的生态作用、通过管理地上枯落物调控陆地生态系统功能和 服务有重要意义。综述了陆地生态系统地上枯落物的积累和分解过程及其影响因素, 然后概括了通过这些过程地上枯落物所 发挥的生态作用,最后,在全球变化背景下,基于当前研究进展提出陆地生态系统地上枯落物研究的前景。地上枯落物累积在 时间尺度上一般遵循植物的生命周期, 同时也受环境因子的调控。大的空间尺度上,枯落物累积主要受水热因子控制,伴随植 被类型的变化, 表现随纬度升高而减少的趋势。然而, 在局域尺度内,枯落物累积除受水、热因子限制,还被群落结构、土壤条 件、植食动物等因素影响, 表现较大变异性。当前, 人类干扰作为一个不可忽视的因素,正在强烈甚至不可逆转的改变地表植被 覆盖和枯落物累积。地上枯落物的分解过程包括淋溶、光降解、土壤动物和微生物分解,这些过程同时进行并相互影响。尽管 目前还不清楚,但区分这些分解过程和分解产物的去向对了解陆地生态系统物质循环有重要意义。枯落物分解首先被自身类 型、化学组成、物种多样性决定, 同时也受分解者群体、非生物环境影响。其中,枯落物分解与其化学特性、物种多样性及土壤养 分状况的关系是研究的热点,也是广泛争议的焦点。通过累积和分解,地上枯落物对陆地生态系统有物理、化学、生物作用。目 前, 枯落物的物理和化学作用研究较为透彻, 而由于受枯落物数量、环境条件、响应植物特征或一些有待挖掘的未知因素的共同 限制, 地上枯落物的生物作用, 尤其对植物的作用在不同研究中仍没有达成普遍的共识。全球变化可能影响地上枯落物累积、 分解和生态作用。在全球变化的背景, 研究地上枯落物产量和性状变化、阐明枯落物分解的分室模型、继续分析枯落物性状和 分解关系、深人揭示枯落物的生态作用及其制约因素, 理解和预测地上枯落物数量和质量变化对陆地生态系统功能和服务的影 响是必要的。
\end{abstract}

关键词:地上枯落物;陆地生态系统;枯落物累积;枯落物分解; 全球变化

\section{The accumulation, decomposition and ecological effects of above-ground litter in terrestrial ecosystem}

\footnotetext{
LI Qiang ${ }^{1,2}$, ZHOU Daowei ${ }^{1, *}$,CHEN Xiaoying ${ }^{1,2}$

1 Northeast Institute of Geography and Agroecology, Chinese Academy of Sciences, Changchun 130102, China

2 University of Chinese Academy of Sciences, Beijing 100049, China
}

\begin{abstract}
Understanding of the accumulation and decomposition processes of above-ground litter is of importance for us to study its ecological function and service in terrestrial ecosystems. The current paper first reviews the accumulation and decomposition processes of above-ground litter in terrestrial ecosystem and factors that affect these two ecological processes, then summarizes the ecological effects of above-ground litter in terrestrial ecosystem as a result of its accumulation and decomposition. Finally, under the view of global climate change, based on previous research progress, we review the
\end{abstract}

基金项目: 国家重点基础研究发展计划 (2011CB403203)

收稿日期: 2012-11-27; 网络出版日期: 2014-02-27

*通讯作者 Corresponding author.E-mail: zhoudaowei@ neiae.ac.cn 
perspectives of research on above-ground litter in terrestrial ecosystem. On the temporal scale, the accumulation of aboveground litter commonly follows the plant life cycle, and it is also regulated by environment factors, such as climatic conditions, soil temperature and moisture. On the large spatial scale, the accumulation of above-ground litter is primarily controlled by hydrothermal factors, resulting in the change in vegetation type, which show a decreased trend with the elevated latitude. However, on the local scale, apart from the constraints of hydrothermal factors, the accumulation of above-ground litter are also impacted by community structure, soil conditions, activities of herbivores, which shows more variability. Human disturbance is also an important factor that induces change of terrestrial vegetation cover and litter accumulation, and in many cases the changes are even irreversible. The decomposition processes of above-ground litter in terrestrial ecosystem include leaching, photo-degradation, soil animal and microbial decomposition. These decomposition processes occur simultaneously and interactively. Although still it is not well known now, distinguishing these decomposition process and fate of decomposition products with them are of importance for understanding the nutrient recycle in terrestrial ecosystem. Litter decomposition firstly is determined by its type, chemical composition, species diversity, but also affected by decomposer community and abiotic environment factors. Among these factors, the relationships between decomposition and litter chemical traits, species diversity and soil nutrient status are the key focus of this study. Through its accumulation and decomposition, above-ground litter has significant effects on terrestrial ecosystem physically, chemically and biologically. Currently, there are ample studies on the physical and chemical properties of above-ground litters, but there are very few studies on the biological property of above-ground litter, especially for the effects above-ground litters on vegetation that are still controversial as they are limited by complicated factors, including litter quantity, environment condition, the traits from impacted plants, even some unknown factors by now. Global climate change may change the accumulation and decomposition of above-ground litter and their ecological effects on the distribution, growth and traits of terrestrial plants in terrestrial ecosystem, through changes of climate and soil conditions. Under the global climate change, it is necessary to study the litter production and trait change on the spatial scales, expound the compartment model of litter decomposition on the temporal and spatial scales, deeply analyze the relationships between above-ground litter traits and decomposition, and further reveal the ecological effects of above-ground litter when relative limited factors are integrated into analysis, understand and predict the impact of above-ground litter on terrestrial ecosystem function and service under the scenario of the future environment change.

Key Words : aboveground litter; terrestrial ecosystem; litter accumulation; litter decomposition; global change

地上枯落物是指由植物地上部分产生并归还到 地表的所有有机物质的总称 ${ }^{[1]}$, 是连接陆地生态系 统地上和地下生态过程的关键媒介 ${ }^{[2]}$ 。地上枯落物 的分解是陆地生态系统物质循环重要过程 ${ }^{[3-5]}$ 。然 而,地上枯落物对陆地生态系统的意义还不仅于此, 覆盖在地表上的枯落物一方面直接作用于地上和地 下生物体, 如通过遮碍影响植物生长, 通过“食物”供 应影响土壤动物和土壤微生物种类、数量和活动 ${ }^{[2]}$; 另一方面地上枯落物构成了陆地生态系统地下部分 和地上环境间的一个 “缓冲区”, 过滤着地上环境对 土壤的作用, 通过对土壤微气候的调控间接地影响 生态系统结构和功能 ${ }^{[6]}$ 。

地上枯落物对陆地生态系统的作用取决于枯落
物的数量、质量和分解特性 ${ }^{[7-8]}$ 。因此, 了解陆地生 态系统地上枯落物的累积及其分解过程对于我们理 解它的生态作用、通过管理地上枯落物调控生态系 统功能和服务尤为重要。本文综述了陆地生态系统 地上枯落物积累、分解过程及其影响因素, 并概述了 通过这些过程地上枯落物所起的生态作用, 最后, 在 全球变化背景下, 结合当前的研究进展提出地上枯 落物研究的前景。

\section{1 陆地生态系统地上枯落物的累积}

\section{1 地上枯落物的累积规律}

一般地,陆地生态系统地上枯落物主要源于地 上植物组织的自然死亡和调落, 但一些环境外力也 
可能造成植物组织的死亡或脱落形成枯落物。地上 枯落物在时间尺度上的累积受植物生命周期操控, 一般在个体或构件生命末期激增,然而,环境条件的 变化可能影响植物的结构分配、组织更新和寿命 ${ }^{[9]}$, 导致枯落物产量和组分在时间尺度上的变化。地上 枯落物累积在大的空间尺度上存在明显的地带性差 异,主要是由水热条件变化导致的植被类型的差异 决定的。一些学者通过研究致力于揭示枯落物生产 的全球模式,如 Wang 等的研究报道全球陆地生态系 统枯落物的生产量随着纬度的升高而降低 ${ }^{[10]}$, 这个 发现与 Matthews 利用模型估算的规律是大体一致 (图 1 ${ }^{[10-11]}$,与 Vogt 和 Xiong 基于森林生态系统的 研究结果也相似 ${ }^{[12-13]}$ 。然而, 上述研究的地理尺度 宽泛,因此空间上水热条件的较大差异掩盖了小尺 度下的环境异质性。尽管这些研究结论对预测全球 尺度上的枯落物产量具有参考作用, 但往往无法解 释同一气候带下枯落物产量的位点间差异, 因为枯 落物和生产力一样,除受水热因子调控外, 局部的土 壤特性、自然和人为干扰等都对其有影响 ${ }^{[2]}$ 。

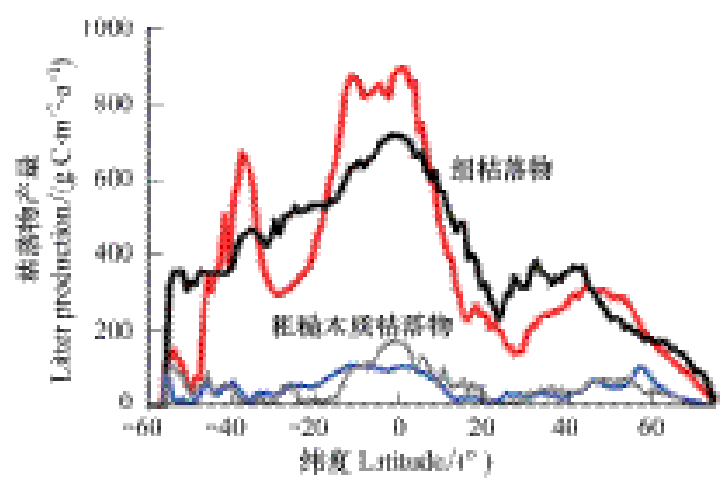

图 1 陆地生态系统枯落物产量随纬度变 [10-11]

Fig.1 The change of litter production in terrestrial ecosystem with latitude ${ }^{[10-11]}$

红色和蓝色线表示 $\mathrm{Wang}$ 等的估计值 ${ }^{[10]}$; 黑色和灰色线表示 Matthews 的估计值 ${ }^{[11]}$

\section{2 决定地上枯落物累积的因素}

\subsection{1 植被类型和群落结构}

水热条件的地理差异决定着陆地生态系统的植 被分布。不同植被类型下的植物在生活史和生活型 上均有所差别, 如森林植被对比草地植被、温带阔叶 林对比寒温带针叶林等。不同植被类型下, 植被地 上生产力往往有差别, 植物器官生长样式和衰老过 程也存在差异, 导致地上枯落物产量的变化, 如张新 平等在中国东北地区森林植被中的 27 个样地调查
发现: 不同森林类型调落物年产量存在显著差异, 针阔叶混交林的地上枯落物年产量显著高于落叶针 叶林和常绿针叶林 ${ }^{[14]}$ 。

在相同的气候条件和植被类型下, 群落的物种 组成和多样性可能通过影响地上生产力决定枯落物 的产量 ${ }^{[15]}$ 。物种组成和多样性对生产力的作用取 决于组成群落各物种的功能特征 (物种组成) 及其关 系 (多样性) 对生产力的贡献。目前解释群落结构和 生产力关系的机制主要有两个假说,一个是“多样性 假说”: 该假说认为群落中有机体间通过资源利用互 补影响生态系统生产力, 支持多样性和生产力存在 正相关关系 ${ }^{[16]}$ 。另一个是 “质量比假说” : 该假说认 为群落结构和生产力关系主要受群落中优势种支 配, 与多样性关系不敏感, 强调物种组成在决定生产 力上的重要性 ${ }^{[17]}$ 。这两个假说被不同的研究所支 持, 但基于研究的位点特异性, 目前为止仍无法评判 哪个理论更具合理性, 因为自然生态系统中, 物种分 布、数量、特征先决于环境条件, 研究位点间环境因 子的差异往往影响群落结构和生产力的关系。

\subsection{2 非生物环境}

大的空间尺度上,水、热、光条件通过控制植被 类型影响地上生产力和枯落物产量。地上生产力和 枯落物产量除受水热光因子影响外, 还受土壤肥力 和理化特性控制, 尤其是在小空间尺度上。这些环 境因子一方面影响物种分布和植物的生长潜力, 一 方面也决定植物的物候特征, 影响植物器官的更新 和衰亡 ${ }^{[2,9]}$ 。Meentemeyer 等基于全球尺度的模型指 出: 在各气候变量中, 实际水分蒸发量能最好的模拟 枯落物产量的变化, 模型指出枯落物产量和地区实 际水分蒸发量正相关。而当把土壤肥力再考虑进这 个模型中, 模型模拟的准确性显著提高, 随着土壤肥 力的增加, 枯落物产量相应提高, 这说明土壤肥力也 影响枯落物的产出 ${ }^{[18]}$ 。Meentemeyer 的模型证明 水、热、肥力因素能很好的解释大的空间尺度上枯落 物产量的差异, 但在小空间尺度上, 可能失去解释能 力, 因为小空间尺度内枯落物的产量的变化更多受 局部环境特征的控制,如 Zhou 等在美国南部大平原 的研究发现枯落物的产量除与大气降水和温度相关 外, 同时受土壤 $\mathrm{pH}$ 值、碳氮比 ( carbon/nitrogen, C/ N) 的影响 ${ }^{[19]}$ 。一些极端的气候因素或环境因子也 会影响到地上枯落物的数量, 如强风、霜冻、冰雨、可 
能促进植物组织的死亡和调落, 自然火的干扰能够 减少地上枯落物的存留 ${ }^{[20-21]}$ 。

\subsection{3 植食动物}

植食动物不断啃食植物地上器官, 能够刺激植 物的补偿性生长 ${ }^{[22]}$, 适当强度的采食下, 地上生物 量可以通过植物器官的物补偿性生长得以弥补, 甚 至增加, 进而提高地上枯落物的产量, 但是当采食强 度过大, 采食行为严重损坏了植物器官的功能, 抑制 了植物器官的再生, 就会降低生态系统地上生产力, 减少地上枯落物的产出 ${ }^{[2]}$ 。植食动物所消费地上生 物量的比例依照不同的生态系统类型也有所差异。 一般在早期弃耕地和林地中, 对植食动物而言, 植物 器官适口性较差, 且植食动物的种类相对较少, 被植 食动物消费的地上生物量所占的比例很小, 绝大部 分地上生物量生产都转化成了枯落物 ${ }^{[3,23]}$ 。而在草 地生态系统中, 植物器官适口性好, 草食动物种类和 数量均较多。由于食草动物的频繁啃食, 大部分的 当年地上生物量都被初级消费者采食掉, 导致地上 枯落物的资源减少, 积聚量显著降低 ${ }^{[24]}$ 。有时, 植 食动物的采食行为 (包括采食、践踏等) 增加了未被 采食的植物器官的损伤和死亡 ${ }^{[25]}$, 导致大量地上生 物量调落到地面形成枯落物, 会在短时间内增加枯 落物的数量。

\subsection{4人为干扰}

随着人口数量的增长, 为满足衣食住行的需要, 人类一方面加剧了对陆地植物资源的索取, 为获取 薪柴和木材,大面积的森林被砍伐, 为满足肉奶产品 的需求,牲畜的饲养量大幅上升,大面积的草地、林 地被过度放牧, 为获取药用植物资源, 陆地植物被大 肆采挖。过度放牧、砍伐和滥采滥挖导致植被的严 重退化。仅就我国,在过去几十年人口迅速膨胀的 压力下, 大面积森林、草地、湿地被过度利用, 天然 林、草地、湿地面积锐减,导致大面积的水土流失、荒 漠化、盐碱化 ${ }^{[26-27]}$, 植被退化促使植被盖度、高度的 下降, 群落结构简单化, 群落功能稳定性下降, 地上 生物量减少,枯落物的产量也将随之降低 ${ }^{[27-28]}$ 。

氮是陆地生态系统地上生产力的关键限制因 子 ${ }^{[29]}$, 为在有限土地上获取更多的收益, 人工氮肥 添加往往被用来提高在陆地生态系统生产力。一般 地, 施肥能够迅速增加植被的地上生产力, 尤其在土 壤退化、养分贫痊的生境中 ${ }^{[30]}$, 短期内, 施肥可能通
过提高生产力间接地增加枯落物数量。但是, 长期 或大量的施肥可能改变植物群落的物种组成, 许多 研究报道氮肥施加降低群落的物种多样性 ${ }^{[31]}$ 。群 落结构的变化相应改变了枯落物的种类组成和质 量, 同时,物种多样性的降低可能导致群落结构和功 能稳定性的下降 ${ }^{[31]}$, 因此, 在长的时间尺度内, 施肥 对群落生产力及枯落物产量的贡献存在不确定性。

除提高天然生态系统的利用强度外, 另一方面 人类正不断地改变着土地利用的方式。如为缓解粮 食、能源、住房和交通压力, 大量的草地和林地、湿地 被转化为农田、矿场、建筑、道路用地 ${ }^{[32]}$ 。如 $\mathrm{Lal}$ 等 估计截至 1998 年,全球约有 $750 \times 10^{6} \mathrm{hm}^{2}$ 的森林被用 作农田, 占土地利用变化的 $45 \%$; 约 $660 \times 10^{6} \mathrm{hm}^{2}$ 的 草地被开层成农田, 占土地利用变化的 $40 \%{ }^{[33]}$ 。土 地利用方式的转变不同程度地改变地表植被覆盖, 甚至彻底移除地上植被, 将严重影响地上的枯落物 数量。

\section{2 地上枯落物的分解}

2.1 地上枯落物的分解过程

地上枯落物的分解过程是枯落物的生物量损失 和化学物质释放的过程 ${ }^{[34]}$, 覆盖在陆地生态系统地 表的枯落物经过水分淋溶、光降解、自然碎化、土壤 动物取食、微生物分解由复杂的有机化合物转化成 简单的有机物和无机化合物 ${ }^{[35-36]}$ 。其中, 淋溶和光 裂解是枯落物在水分和光照作用下的分解过程。在 湿地或者降水频繁的生态系统中, 淋溶是枯落物分 解的一个主要过程 ${ }^{37]}$, 而在干旱或者半干旱的生态 系统中, 降水减少, 淋溶作用减弱, 这时, 光降解对枯 落物分解的影响显著增加。有研究表明: 针对干旱 或半干旱生态系统,或者木质素含量较高而不易被 微生物分解的枯落物, 光降解对枯落物的分解起到 至关重要的作用 ${ }^{[38-39]}$ 。淋溶过程进行的同时,土壤 动物的采食或自然因素将枯落物破碎化, 把大块的 有机物碎解成小块进人土壤被土壤微生物进一步利 用分解。

淋溶、光裂解、土壤动物取食、微生物分解是枯 落物分解过程中物质损失的主要途径, 清楚这些分 解过程和分解产物的去向, 对了解生态系统物质循 环有重要意义。然而实际分解中, 这几个过程同时 发生并相互影响, 如淋溶和光裂解可能影响被微生 
物分解的枯落物的形态和物质组成。自然条件下, 很难区分这些分解过程和分离分解产物, 尽管一些 研究结合特定位点的环境条件, 通过因子添加或去 除实验阐述了参与分解的某一因子的相对作 用 ${ }^{[40-41]}$, 但未见有研究明确阐明每个分解过程对枯 落物分解的贡献及分解产物的动态。

2.2 影响地上枯落物分解的因素

\subsection{1 枯落物的类型}

由于结构和物质组成的差异, 不同类型的枯落 物分解速率也有差别 ${ }^{[42]}$ 。一般, 裸子植物的枯落物 具有较高木质素、纤维和次生代谢产物含量, 较被子 植物枯落物分解慢 ${ }^{[43]}$, 植物幼龄器官由于具有较少 的木质素、纤维及次生代谢物, 因此较老龄器官分解 更快 ${ }^{[2]}$ 。作为支撑器官, 植物枝条或茎往往具有更 高的木质素和纤维素含量, 因此相对光合器官和繁 殖器官可能分解更缓慢 ${ }^{[44]}$ 。枯落物分解是个“古老 的”研究主题,已发表的研究中,一般单独以叶片或 地上总体枯落物作为研究对象, 尤以植物叶片枯落 物的分解过程被研究较为详细, 但关于植物枝条、 茎、花、果等枯死组织分解特性的数据支持较少 ${ }^{[44]}$, 有待更深人的研究。

\subsection{2 枯落物物质组成与含量}

枯落物物质组成和含量一方面决定其分解过程 和环境因子的关系,如可溶性成分高的枯落物可能 具有更强的水分淋溶作用, 高木质素含量枯落物的 分解对光的依赖性更强 ${ }^{[45-46]}$, 另一方面, 枯落物物 质组成及其含量决定分解微生物的喜好程度, 因此 决定其微生物分解速率 ${ }^{[47]}$ 。

基于和枯落物分解速率的相关性, 目前被广泛 用于评价枯落物质量的变量包括 $\mathrm{C} 、 \mathrm{~N}$ 、磷 $(\mathrm{P})$ 、木质 素 (lignin) 含量、 $\mathrm{C} / \mathrm{N}$ 、 木质素与氮元素含量比 $(\operatorname{lignin} / \mathrm{N})$ 、氮磷比 $(\mathrm{N} / \mathrm{P})$ 等。一般地, 高 $\mathrm{N} 、 \mathrm{P}$ 含 量, 低木质素含量被作为枯落物高质量的标志。一 些研究支持枯落物中氮含量和枯落物分解速率呈现 正相关关系，而木质素含量对枯落物分解有负效 应 ${ }^{[48-50]}$ 。Zhang 等总结已发表研究数据发现全球尺 度内, 枯落物的 N、P 的含量越高, 枯落物分解速率越 快, 木质素含量、lignin $/ \mathrm{N} 、 \mathrm{C} / \mathrm{N}$ 与枯落物分解速率的 关系为负相关 ${ }^{[51]}$ 。虽然大量研究支持枯落物分解 速率和上述特征的相关关系, 也有一些研究对这些 关系提出质疑和补充。如 Strickland 等发现高质量
的草本枯落物在森林生境下并未表现较木本植物枯 落物更快的分解速率 ${ }^{[47]}$ 。他提出枯落物化学特性 在分解过程中的决定作用依赖于其分解者对这些化 学特征的敏感性, 独特类群的微生物可能专一性偏 爱某一种化学成分或化学成分之间的比例关系, 而 对其它化学特征并不偏爱, 甚至抵触, 因此, 具备高 质量并不意味着枯落物就具有高的分解速率。 $\mathrm{Li}$ 等 发现枯落物分解早期, 分解速率与枯落物中氮含量 正相关, 而在后期分解过程中, 分解速率与枯落物中 氮含量却呈现负相关 ${ }^{[52]}$, 这个发现与 Berg 和 Matzner 综述中的结论一致 ${ }^{[53]}$, 这是因为在不同的分 解阶段, 枯落物的分解速率可能受不同的化学组分 限制, 如有研究者提出在分解早期, 枯落物 $\mathrm{N}$ 含量是 分解的主要限定因素, 而在分解后期, 枯落物的分解 主要受木质素含量的影响 ${ }^{[54]}$ 。因此, 研究中评估枯 落物质量和分解关系的时间尺度可能影响最后的结 论。枯落物分解不仅受其化学特征影响, 局域的微 气候环境也制约其分解过程 ${ }^{[55]}$ 。不同的地域条件 下, 受气候、土壤微环境条件的限制, 分解生物对枯 落物的分解能力可能有差异, 导致枯落物质量和分 解速率关系的变化。

除上述变量外,枯落物中的一些金属元素也影 响分解过程。如 Zhang 等的研究表明枯落物分解和 钾、钙、镁的含量正相关 ${ }^{[51]}$ 。最近的研究表明金属 元素在枯落物分解中的作用主要是参与分解酶的合 成和激活, 如 Berg 等研究发现, 针叶叶片枯落物的 分解速率对枯落物中锰元素的含量有很强的依赖 性, 因为锰是枯落物分解晚期木质素分解酶的关键 组成成分, 锰含量越高, 枯落物分解则越快 ${ }^{[56]}$ 。自 然界中,一些植物基于生理特性或在环境刺激下合 成了大量次生代谢产物, 这些次生代谢物往往是高 度化合或具有化学毒性的, 不仅自身很难分解, 有的 次生代谢如单宁 (Tanin) 等, 还可能对土壤微生物有 毒害作用, 降低微生物的分解利用 ${ }^{[57]}$ 。

\subsection{3 物种多样性}

过去的 20a, 物种多样性和生态系统功能之间的 关系一直是生态学家们研究的热点,包括产量形成、 碳氮循环等已经被证实和物种多样性存在紧密的联 系 ${ }^{[15,58-59]}$ 。枯落物分解是植物多样性影响地下生态 系统养分循环的一个主要途径, 其和物种多样性的 关系近来也被广泛研究 ${ }^{[60-61]}$ 。物种多样性增加后, 
混合枯落物的分解速率往往不同于基于单物种分解 速率和其在混合枯落物中比重计算的预期值, 这种 现象被称之为“非加性效应(no-additive effect)”。这 种多样性效应提出后已经被许多研究检验, 并且多 数研究支持物种多样性对枯落物分解有正的 “非加 性效应” ${ }^{[48,54]}$, 同时, 相关的机制被提出来解释这种 现象。首先,多种枯落物在大小、形态上的差异可能 为土壤动物创造多样化的生境, 也增加了食物网的 复杂程度, 可能增加土壤动物的多样性和数量。其 次,一些研究认为土壤中的微生物与枯落物的化学 物质具有特异性的联系, 单一物种枯落物往往导致 低的微生物种类多样性, 而混合枯落物增加了物质 组成的丰富度, 可能增加微生物种类多样性。再次, 物种多样性带来的枯落物化学物质组成的差异可能 促进养分从易分解枯落物向难分解枯落物的转移, 间接增加枯落物的分解速率 ${ }^{[61]}$ 。也有研究观察到 物种多样性对枯落物分解的负的 “非添加效应”, 如 McArthur 等在一个河滩生态系统的混合枯落物分解 实验中发现两种柇树和枫树枯落物混合后分解速率 较预期值速率低 ${ }^{[62]}$,类似的结论被 Nilsson 等在一种 灌木和苔藓枯落物的混合分解实验中报道 ${ }^{[63]}$ 。通 过对这些产生“负效应” 的个例中, 发现混合枯落物 对分解负作用的产生往往与研究位点所涉及的物种 的特异性有关。一些研究位点中, 被混合的枯落物 往往包括富含次生代谢物的物种, 这些物种不仅自 身分解缓慢，还可能通过此生代谢产物的释放控制 土壤微生物影响其他物种的分解。因此,组成混合 枯落物物种组成和功能特征对预测分解能力非常重 要。另有研究发现物种多样性增加并未导致枯落物 分解速率与预期值之间的差异 ${ }^{[64]}$ 。这样的结果可 能有两种产生方式:其一,尽管被混合在一起,但每 个物种的分解过程是相互独立的, 分解过程中未相 互影响; 其二, 混合的物种分解过程中存在相互作 用, 但这种相互作用此消彼长, 对总体枯落物分解速 率无影响, Lorenzen 等的研究支持这个解释 ${ }^{[65]}$ 。通 过上述总结, 发现多样性与枯落物分解关系还有很 多不确定性,两者之间关系的机理研究还不够深人。 物种多样性本身牵涉物种组成、数目和均匀性, 这其 中究竟哪些因子控制着物种多样性和枯落物分解之 间的关系? 另外,其他因素如气候、土壤条件等是否 影响两者关系? 这些问题都还有待深人探讨。

\subsection{4 分解者}

生态系统的分解者包括土壤动物和土壤微生 物。而在许多枯落物分解研究中, 尤其是通过网袋 法或室内培养法进行的研究中, 土壤动物对分解的 作用往往被忽略了。土壤动物对枯落物分解的贡献 主要体现在加速分解进程和促进土壤微生物活 动 ${ }^{[66-67]}$ 。首先, 土壤动物能够采食枯落物, 一方面将 大块的枯落物破碎化, 同时通过排泄将难分解的枯 落物转化成容易分解的物质 ${ }^{[68]}$ 。再者, 土壤动物掘 洞、食物搬运和储藏等行为促进了植物残体和土壤 微生物的接触 ${ }^{[69]}$ 。其三, 土壤动物死亡残体本身为 土壤微生物提供了食物。最后, 土壤动物的活动改 善了土壤微生物的生存条件, 如降低土壤容重, 改善 土壤的水、气状况等 ${ }^{[66-67]}$ 。因此, 土壤动物在枯落物 的分解过程中应占有很重要的角色, 如 Anderson 的 研究就表明土壤动物能显著够加速阔叶枯落物的破 碎速度 ${ }^{[69]}$, Verhoef 和 Brussaard 报道土壤动物对包 括森林、农田、草地生态系统在内的枯落物氮的分解 平均贡献率为 $30 \%{ }^{[70]}$ 。

参与枯落物分解的土壤微生物主要是细菌和真 菌 ${ }^{[71-72]}$ 。真菌通过分泌酶的作用能进入死亡植物器 官内部, 利用其中养分进行繁殖、生长, 将死亡植物 降解; 个体较小的细菌, 可以存活于真菌活动所产生 的空隙中, 当营养物质供应充足时, 它们会快速生长 和繁殖, 加快死亡植物材料的分解 ${ }^{[71]}$ 。有研究发 现, 暴露于空气中的枯落物碎片主要被真菌利用分 解 ${ }^{[2]}$, 而浸在水中的枯落物碎片主要被细菌分解 掉 ${ }^{[37]}$ 。伴随着地表和土壤水分含量的变化, 真菌和 细菌的数量可能会发生相应的变化, 影响枯落物的 分解。

分解者的数量和种类决定着枯落物的分解速 率 ${ }^{[66]}$ 。分解者数量的增加提高了对枯落物的采食 量, 而分解者种类的增加会增加彼此间的相互作用, 如一些节肢动物、蚯蚓等可以碎化大块有机体供土 壤微生物利用 ${ }^{[66]}$ 、真菌能够帮助细菌进人死亡植物 组织内部 ${ }^{[73]}$, 多种微生物可以提供更多功能的分解 酶, 这会补偿单一种类酶分解能力的不足, 促进枯落 物的分解过程 ${ }^{[74]}$ 。

\subsection{5 非生物环境因子}

大的空间尺度下, 温度和降水是影响枯落物分 解的主要非生物环境因子 ${ }^{[55]}$ 。以往的研究发现高 
温可以促进枯落物的分解, 反之, 低温则抑制枯落物 的分解 ${ }^{[2,12]}$, 这是因为多数微生物适合在较高温度 下生活, 合适的温度可以促进微生物群体的活力, 增 进分解酶的活性, 加快枯落物的分解。降水是影响 枯落物分解的另一重要因子, 降水不仅在枯落物的 淋溶阶段起着重要的作用,也通过土壤水分的调节 也直接决定地下生物群体的生存环境, 影响地下生 物种类、数量和活力, 进而影响到枯落物的分解 ${ }^{[2]}$ 。 土壤水分含量过低对地下生物的生存和繁殖往往是 不利的,而水分含量过高又会影响土壤的通气情况， 也不利于土壤生物生存 ${ }^{[55]}$ 。在干旱或半干旱生态 系统中,水分因素可能成为限制枯落物分解的主要 因子 ${ }^{[75]}$, 即使在降水量高的地区, 水分的季节性变 化导致的阶段性干旱也会限制枯落物的分解 ${ }^{[76]}$ 。 降水也可能能通过影响植物的生长和生物量在地上 和地下的分配间接影响土壤特征和地下生物的食物 数量和构成,进而影响分解者 ${ }^{[19]}$ 。温度和水分在时 间尺度上的变化导致的土壤的干湿交替和冻融现象 往往促进枯落物分解。一方面,这两种现象可能加 速枯落物的裂解程度 ${ }^{[35,37]}$; 另一方面, 土壤水分和温 度状况的交替转变可能刺激微生物的活性, 如有研 究者报道土壤干燥或结冻以后, 土壤微生物大量死 亡, 当土壤重新湿润或解冻后, 存活的土壤微生物就 会利用死亡的微生物残体作为能源, 其活力可能被 激发,土壤的反复冻融可能打破土壤有机质受团粒 的物理保护, 增加土壤微生物的分解基质, 可能提升 其数量和活动 ${ }^{[77-78]}$ 。

除温度和降水外,枯落物分解还受土壤养分的 影响 ${ }^{[48,52]}$ 。土壤养分是满足微生物生长需要的能量 来源; 同时,土壤养分也影响植物组织的化学组成和 含量, 因此土壤养分可能是影响微生物分解枯落物 的关键因素。一些研究认为增加土壤养分导致微生 物生物量和枯落物质量的提高可能加快枯落物分解 的速率 ${ }^{[79]}$, 然而, 另外一些研究发现土壤养分的增 加并不加速枯落物分解, 甚至延缓枯落物分解 ${ }^{[00-81]}$ 。 自然生态系统中, 植物死亡进人土壤有机质库, 再由 微生物分解掉, 释放的养分再被植物吸收用于构建 有机体。通过这个物质循环链, 在植物、土壤、土壤 微生物间应存在着严格的化学计量学上的联系 ${ }^{[82]}$ 。 换句话说, 在某一特定位点, 土壤微生物个体的构建 表现特定的化学计量特征, 相似的化学计量关系也
将体现在植物和土壤中。这种通过化学计量学构建 的三者稳定关系形成于长期的植土互作下,土壤养 分的变化改变了生态系统原来的土壤、植物的化学 计量特征,这种变化需要微生物群体做出适应和调 整, 如果微生物的调整是积极的, 就可能增加枯落物 的分解, 反之, 就可能抑制分解, 这可能是土壤养分 对枯落物分解被报道有不同作用的原因。除土壤养 分外, 土壤的通气状况、 $\mathrm{pH}$ 也能通过影响微生物的 生命活动影响枯落物的分解 ${ }^{[83]}$

相同的气候和土壤条件下,一些干扰因素也可 能造成小尺度的环境差异,也会影响枯落物的分解。 如在枯落物分解过程中, 某些外力因素如地表碾压、 土壤翻动等可能加速枯落物破碎或与使其土壤接触 更加紧密, 往往会增加枯落物的分解速率 ${ }^{[84]}$ 。火的 发生能够导致土壤温度、水分、生物群体的迅速变 化 ${ }^{[85]}$, 还可能影响植被的化学计量特征决定枯落物 质量 ${ }^{[86]}$,进而影响枯落物的分解。

\section{3 地上枯落物在生态系统中的作用}

地上枯落物在生态系统中扮演着两个关键的角 色。首先, 枯落物分解生态系统物质循环的关键过 程; 其次,枯落物构成了地表的一个保护层, 通过它 的遮蔽作用调节土壤的微气候条件, 直接或间接影 响植被生长、地下生物活动及地下生态过程。枯落 物在生态系统中的角色可体现在物理、化学、生物作 用 3 个方面。

\section{1 物理作用}

覆盖于地表的枯落物可以降低地表温度、减少 土壤水分蒸发、起到保持土壤水分含量的作用 ${ }^{[2]}$, 然 而,大量枯落物的聚集也可能截留大气降水,对土壤 水分有负效应 ${ }^{[87]}$ 。枯落物的覆盖避免了外界环境 和土壤表面的直接接触, 减弱了外界干扰对土壤和 地面植被的冲击, 可以减缓风水侵蚀、动物践踏等对 土壤的破坏。枯落物的分解增加了土壤腐殖质含 量, 有利于土壤团粒的构建, 也能起到降低容重、松 软土壤的作用 ${ }^{[6]}$ 。然而, 枯落物的积累也造成土壤 表面光透射的减少，导致近地面植物可利用光辐射 的降低 ${ }^{[88]}$ 。枯落物的覆盖使土壤表面的种子避免 暴露在地面上,从而减少了种子被捕食的几率,但同 时, 当植物种子散落到地表枯落物上时, 由于枯落物 层的隔离使得一些种子无法立即接触到土壤, 就会 
延缓种子发芽时程, 降低种子发芽的成功率 ${ }^{[89]}$ 。再 者, 在枯落物的阻碍作用下, 即使幼苗成功出土, 植 物幼苗的延展也会受到抑制, 影响生长 ${ }^{[90]}$ 。

\section{2 化学作用}

地上枯落物的存在对土壤的养分动态有强烈的 作用。一方面枯落物的分解是陆地生态系统土壤养 分回还的关键过程, 另一方面枯落物也通过其对土 壤微环境的调控间接地影响到土壤的养分循环过 程 ${ }^{[6]}$ 。枯落物质量与其降解速率和土壤的养分回收 能力有直接的关系, 其不仅决定输人土壤的养分组 成和数量 ${ }^{[91]}$, 也能够通过自身的性质影响土壤生物 的活性,进而影响土壤中有机物质的分解 ${ }^{[59-60]}$ 。枯 落物的输人和腐殖化增加了土壤中有机质的含量, 分解缓慢的枯落物能够增加土壤有机质库的长期稳 定性,然而养分含量高、易分解的枯落物往往对土壤 微生物活性有激发效应( Prime effect), 可能诱发土 壤原有有机物质矿化速率的提高, 反而影响土壤有 机质的累积,在一些利用土壤接种培养、通过碳释放 量估计枯落物分解的研究中, 研究人员发现碳的实 际释放量远远超过枯落物的损耗量, 这说明枯落物 的输人刺激了土壤有机质的分解 ${ }^{[92]}$ 。枯落物在土 壤表面的累积对土壤的温度、水分有强烈地调节作 用,借此能够间接地影响土壤生物的生存环境并影 响其数量和活动, 进而影响有机物质的分解和养分 的释放、转移、利用 ${ }^{[93]}$ 。枯落物的分解作用还能够 影响到土壤的酸碱性质 ${ }^{[6,94]}$ 某些盐生植物枯落物的 分解还可以增加土壤中盐分的积聚 ${ }^{[2]}$, 除此之外, 枯 落物中所富含的各种离子和化学物质也会通过其分 解在土壤中富集,包括某些重金属离子、众多的次生 代谢产物包括植物毒素 ${ }^{[94]}$ 。

\section{3 生物作用}

枯落物对生态系统物理和化学作用的生态效应 最终将通过生物作用得以体现。地上枯落物是土壤 中屑食生物和腐食生物的食物来源, 为土壤生物的 生长和繁殖提供能量, 其数量和质量直接相关于土 壤生物类群组成和生物量 ${ }^{[6]}$, 有些枯落物分解过程 会产生的一些次生代谢物,这些次生代谢物一般具 有较低的微生物利用率, 有些物质还可能对土壤微 生物有毒害作用, 如一些枯落物中含有的单宁物质 能与蛋白结合抑制机体对蛋白的利用和酶的活性, 影响其生长和繁殖 ${ }^{[57]}$ 。自然生态系统中, 通过枯落
物分解过程和化学计量联系, 植物-土壤-土壤生物长 期互作,可能在区域尺度上形成特异性的植被、土壤 和土壤生物特征 ${ }^{[83,95]}$ 。除为土壤生物提供食源外, 枯落物对土壤条件的调控能够影响土壤生物的生存 环境, 如枯落物改善土壤水分、降低土壤容重增加土 壤透气性、提供多样化的生境,这些因素可能促进土 壤生物的活动、增加其数量和多样性 ${ }^{[2]}$, 而土壤生物 的活动也将反馈影响土壤养分动态和理化条件。

植物是地上枯落物影响的最重要对象。首先, 枯落物覆盖本身直接影响植物种子的位置, 当植物 种子被枯落物覆盖时, 其保护作用可能增加发芽的 种子数, 而当种子和土壤间被枯落物隔绝, 使其不能 及时接触土壤, 就可能延缓种子发芽, 同时也增加了 种子被捕食的几率, 这对植物建植是不利的 ${ }^{[2]}$ 。其 次, 枯落物覆盖可能阻碍幼苗个体的延伸, 阻碍其生 长 ${ }^{[6,90]}$ 。除了直接的作用外, 枯落物的覆盖、分解对 温、水、光、土壤理化特性等环境因子的调节对植物 有间接的影响,可能改变物种间的竞争格局、影响植 物的地上和地下部分生长、群落结构和生产力 ${ }^{[13]}$ 。 地上枯落物对植物作用的大小、方向取决于枯落物 对植物的促进和限制作用间的平衡 ${ }^{[8]}$, 而这种平衡 关系主要受枯落物数量和质量、环境条件、物种特性 等因素影响。适宜数量的枯落物覆盖可能通过保护 种子、改善土壤水分促进植物建植, 而当大量的枯落 物聚集在地表时, 受限的光资源和阻碍作用对植物 生长的抑制将占据主要。如 Loydi 等综合草地系统 的研究结果得出: 当地表枯落物数量少于 $500 \mathrm{~g} / \mathrm{m}^{2}$ 时能够促进植物幼苗建植, 而当地表枯落物量大于 $500 \mathrm{~g} / \mathrm{m}^{2}$ 时就会抑制植物幼苗建植 ${ }^{[96]}$ 。环境因子也 影响枯落物对植物的作用, 如在水分受限的生态系 统中, 枯落物对土壤水分的积极调节可能掩盖其不 利作用促进植物生长, 而当水分非植物生长限制因 子的, 枯落物就可能表现对植物的负作用 ${ }^{[97]}$ 。植物 自身特征也决定其对枯落物的响应。有研究指出, 枯落物覆盖下, 大种子植物较小种子植物具有更好 的适应性, 因为大种子拥有更多的物质储存, 保证其 具有更强的出土能力, 并在光资源限制环境下保证 幼苗的养分供应 ${ }^{[97-98]}$, 尽管多数研究支持这个结论, 也有一些研究对其提出质疑和补充, 虽然大种子植 物具有优越的萌发和生长潜力, 但也可能增加其调 落的种子被枯落物阻隔的机会, 而且在枯落物作用 
下,植物也能通过生活史策略的调节适应枯落物的 影响, 不同的研究背景下,大小种子对枯落物覆盖的 表现可能有所变化 ${ }^{[8]}$ 。实际研究中,多个因素都可 能导致枯落物对植被作用的变化。Xiong and Nilsson 综合大量研究的数据统计得出: 枯落物对陆地植被 更倾向于有负效应,但不同研究中, 枯落物对植物发 芽、建植、物种丰富度和地上生物量的作用大小不 同。对这 4 个变量, 生态系统类型、枯落物类型、目 标物种、研究方法、研究期限等因素对枯落物作用变 化的解释能力共为 $18.1 \%$ - 39.29\%（图 2), 包括地 理纬度、枯落物数量,还有许多未知因素则解释了枯 落物效应变化的 $60 \%$ 以上 ${ }^{[13]}$ 。Loydi 等最近的研究 中指出: 在草地生态系统中, 研究方法、草地类型、水 分条件、枯落物数量和种子大小等对植物建植响应 枯落物的程度和方向均有影响 ${ }^{[96]}$ 。由上可见, 枯落 物的生物效应受自身和外界多个因子复杂调控, 隐 藏在枯落物作用背后的机制和规律还不清楚, 值得 更深人的研究。

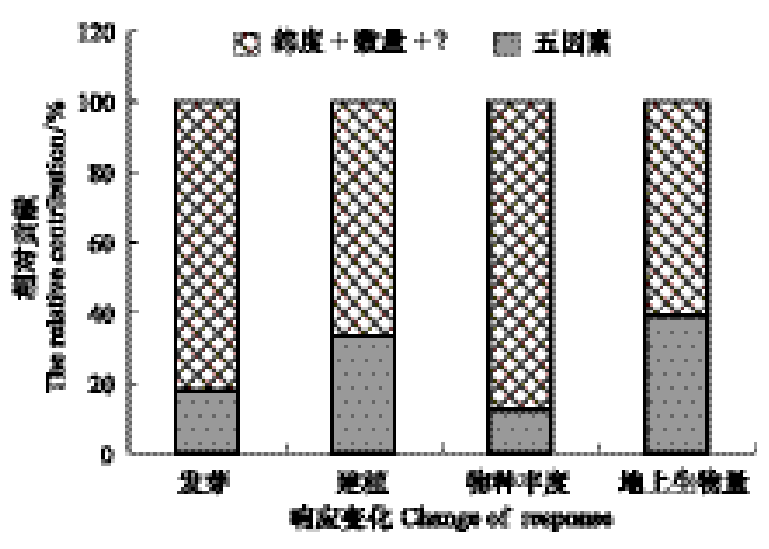

图 2 不同因素对枯落物作用差异的相对贡献

Fig.2 The relative contribution of different factors to difference of litter effects

五因素; 生态系统类型+枯落物类型+作用物种+研究方法+研究 期限;?: 未知因素;数据收集自 Xiong 和 Nilsson ${ }^{[13]}$

\section{4 全球变化背景下枯落物研究重要性和研究展望}

伴随着人类工业的迅猛发展和人口数量的急剧 增加, 大量的燃料燃烧导致温室气体排放、氮沉降的 增加,加之人类为满足生存需要而对自然生态系统 的大规模人为改造,使得整个生物圈正面临全球变 化的挑战。全球变化一方面打破了生物圈各层面或 区域间原有的温度、降水、氮分布格局, 并由此将改 变植物的分布、数量和特征。由于各生态因子在生
态系统内的紧密联系, 个别因子的变化可能在整个 生态系统内产生“连锁反应”。在全球变化下, 原有 的生态系统秩序可能被“重新洗牌”, 原有的生态过 程、功能、服务,包括枯落物累积、分解和生态作用也 可能发生变化, 全球变化为传统的生态学研究提供 了新的视角。

以往研究对枯落物的产生、分解和生态作用已 展开了深人的探讨, 尤其枯落物分解过程及其制约 因素、枯落物对土壤环境及植被的作用被给予了更 多的研究。由于枯落物与气候、土壤、植物、土壤生 物之间的紧密联系,而全球变化可能影响这些因子、 如全球变化影响土壤温度、水分、养分、影响植物生 长和性状、影响植物和地下生物群的物种多样性, 这 些因素的变化将影响枯落物与环境、生物的作用关 系并进一步影响它的生态作用。鉴于全球变化的背 景和当前的研究进展, 关于枯落物研究, 以下是应继 续深人探讨的内容: (1) 空间尺度上, 全球变化诱导 的环境因子改变对地上枯落物的产量、组成、性状的 影响。(2) 空间和时间尺度上, 研究环境因子 (如 光、温、水、基质条件)、分解者群体 (土壤动物、真菌、 细菌等) 对地上枯落物分解的决定作用及相互关系, 建立枯落物分解的分室模型, 预测未来区域环境变 化对地上枯落物分解的影响。(3)物种组成、性状组 成对枯落物分解的影响, 剖析单一物种、单一性状及 物种组合和性状组合对枯落物分解的决定作用。 (4) 围绕枯落物分解过程和化学计量特征构建的植 物-枯落物-土壤微生物-土壤-植物的关系链对未来 环境变化的响应。(5) 枯落物数量、种类、物质组成 对植被的影响和机理及其环境依赖性 (6) 环境变化 诱导的枯落物覆盖、分解变化对未来生态系统功能 和服务的影响, 如水热调控、物质循环、群落构建、生 态系统生产力等。

致谢 感谢澳大利亚 Wagga Wagga 农业研究所李光 棣研究员对写作的帮助。

\section{References:}

[ 1 ] Odum E P. Fundamentals of Ecology. Philadelphia and London: W B Saunders Company, 1971: 109-121.

[ 2 ] Facelli J M, Pickett S T A. Plant litter: its dynamics and effects on plant community structure. Botanical Review, 1991, 57(1): $1-32$.

[ 3 ] Odum E P. Organic production and turnover in old field 
succession. Ecology, 1960, 41( 1) : 34-49.

[ 4 ] Berg B, McClaugherty C. Nitrogen and phosphorus release from decomposing litter in relation to the disappearance of lignin. Canadian Journal of Botany, 1989, 67(4) : 1148-1156.

[ 5 ] Sun O J, Campbell J L, Law B E, Wolf V. Dynamics of carbon stocks in soils and detritus across chronosequences of different forest types in the Pacific Northwest, USA. Global Change Biology, 2004, 10(9) : 1470-1481.

[ 6 ] Sayer E G. Using experimental manipulation to assess the roles of leaf litter in the functioning of forest ecosystems. Biological Reviews, 2006, 81(1): 1-31.

[ 7 ] Amatangelo K L, Dukes J S, Field C B. Responses of a California annual grassland to litter manipulation. Journal of Vegetation Science, 2008, 19(5) : 205-212.

[ 8 ] Hovstad K A, Ohlson M. Physical and chemical effects of litter on plant establishment in semi-Natural grasslands. Plant Ecology, 2008, 196(2): 251-260.

[ 9 ] Zhan F, Yang D M. Relationships among light conditions, crown structure and branch Longevity: a case study in Osmanthus fragrans and Metasequoia glyptostroboides. Acta Ecologica Sinica, 2012, 32(3) : 984-992.

[10] Wang Y P, Law R M, Pak B. A global model of carbon, nitrogen and phosphorus cycles for the terrestrial biosphere. Biogeosciences, 2010, 7(7): 2261-2282.

[11] Matthews E. Global litter production, pools, and turnover times: estimates from measurement data and regression models. Journal of Geophysical Research, 1997, 102(15): 18771-18800.

[12] Vogt K A, Grier C C, Vogt D J. Production, turnover, and nutrient dynamics of above-and below-ground detritus of world forests. Advances in Ecology Research, 1986, 15: 303-377.

[13] Xiong S J, Nilsson C. Dynamics of Leaf litter accumulation and its effects on riparian vegetation: a review. The Botanical Review, 1997, 63(3) : 240-264.

[14] Zhang X P, Wang X P, Zhu B, Zong Z J, Peng C H, Fang J Y. Litter fall production in relation to environmental factors in northeast China's forests. Acta Phytoecologica Sinica, 2008, 32 (5) : 1031-1040.

[15] Tilman D, Reich P B, Knops J, Wedin D, Mielke T, Lehman C. Diversity and productivity in a long-term grassland experiment. Science, 2001, 294(5543) : 843-845.

[16] Tilman D. Distinguishing between the effects of species diversity and species composition. Oikos, 1997, 80(1): 185.

[17] Grime J P. benefits of plant diversity to ecosystems: immediate, filter and founder effects. Journal of Ecology, 1998, 86 (6) : 902-910.

[18] Meentemeyer V, Box E O, Thompson F L. World patterns and amounts of terrestrial plant litter production. BioScience, 1982, $32(2): 125-128$

[19] Zhou X H, Talley M, Luo Y Q. Biomass, litter, and soil respiration along a precipitation gradient in southern great plains, USA. Ecosystems, 2009, 12(8) : 1369-1380.

[20 ] Conner W H, Day J W. Productivity and composition of a baldcypress-water tupelo site and a bottomland hardwood site in a Louisiana swamp. American Journal of Botany, 1976, 63 (10): 1354- 1364.

[21 ] Peterson D L, Rolfe G L. Nutrient dynamics and decomposition of litterfall in floodplain and upland forests of central Illinois. Forest Science, 1982, 28(4): 667-681.

[22] Dyer M I, Turner C L, Seastedt T R. Mowing and fertilization effect on productivity and spectral reflectance in Bromus inermis plots. Ecological Applications, 1991, 1(4) : 443-452.

[23] Whittaker R H, Woodwell G M. Structure, production and diversity of the oak-pine forest at Brookhaven New York. Journal of Ecology, 1969, 57(1): 157-174.

[24] Knapp A K, Seastedt T R. Detritus accumulation limits productivity of tallgrass prairie. BioScience, 1986, 36 ( 10$)$ : 622- 668 .

[25] Choudhury D. Herbivore induced changes in leaf-litter resource quality: A neglected aspect of herbivory in ecosystem nutrient dynamics. Oikos, 1988, 51(3): 389-393.

[26] Li B. The rangeland degradation in north China and its preventive strategy. Scientia Agricultura Sinica, 1997, 30(6) : 1-9.

[27] Liu G H, Fu B J, Chen L X, Guo X D. Charzcteristics and distributions of degraded ecological types in China. Acta Ecologica Sinica, 2000, 20(1): 13-19.

[28] Pei S F, Fu H, Wan C G. Changes in soil properties and vegetation following exclosure and grazing in degraded Alxa desert steppe of Inner Mongolia, China. Agriculture, Ecosystem and Environment, 2008, 124(1/2): 33-39.

[29] Vitousek P M, Howarth R W. Nitrogen limitation on land and in the sea: how can it occur?. Biogeochemistry, 1991, 13 (2): 87- 115 .

[30 ] Frink C R, Waggoner P E, Ausubel J H. Nitrogen fertilizer: retrospect and prospect. Proceedings of the National Academy of Sciences of the United States of America, 1999, 96 ( 4): 1175-1180.

[31 ] Suding K N, Collins S T, Gough L, Clark C, Cleland E E, Gross K L, Milchunas D G, Pennings S. Functional- and abundancebased mechanisms explain diversity loss due to $\mathrm{N}$ fertilization. Proceedings of the National Academy of Sciences of the United States of America, 2005, 102(12): 4387-4392.

[32 ] Meyer W B, Turner II B L. Human population growth and global land-use/cover change. Annual Review of Ecology Systematics, $1992,23(1): 39-61$.

[33] Lal R, Follett R, Stewarteds B A. Management of Carbon Sequestration in Soil. Boca Raton: CRC Press, 1998: 1- 10.

[34] Mun H T. Weight loss and nutrient dynamics during leaf litter decomposition of Quercus mongolica in Mt. Worak National Park. 
Journal of Ecology and Field Biology, 2009, 32(2) : 123-127.

[35] Chapin F S, Matson P A, Mooney H A. Terrestrial Decomposition in Principles of Terrestrial Ecosystem Ecology. New York: Springer Science, Business Media Press, 2002: 151-175.

[36] Brandt L A, King J Y, Hobbie S E, Michunas D G, Sinsabaugh $\mathrm{R}$ L. The role of photodegradation in surface litter decomposition across a grassland ecosystem precipitation gradient. Ecosystems, $2010,13(5): 765-781$.

[37] Polunin N V C. The decomposition of emergent macrophytes in fresh water. Advances in Ecological Research, 1984, 14: $115-166$.

[38] Austin A T, Vivanco L. Plant litter decomposition in a semiarid ecosystem controlled by photodegradation. Nature, 2006, 442 (7102) : 555-558.

[39] Gallo M E, Porras-Alfaro A, Odenbach K J, Sinsabaugh R L. Photoacceleration of plant litter decomposition in an arid environment. Soil Biology and Biochemistry, 2009, 41 ( 7 ): 1433-1441.

[40] Day T A, Zhang E T, Ruhland C T. Exposure to solar UV-B radiation accelerates mass and lignin loss of Larrea tridentate litter in the Sonoran Desert. Plant Ecology, 2007, 193(2) : 185-194.

[41] Matsumoto S, Yusnaini S, Niswati A. Relation to soil animal populations and plant litter decomposition in a tropical region. Edaphologia, 2000, 65(3) : 55-60.

[42] Berg B, Berg M P, Bottner P, Box E, Breymeyer A, Ca de Anta R, Coutaeux M, Escudero A, Gallardo A, Kratz W, Madeira M, Mälkönen E, McClaugherty C, Meentemeyer V, Muñoz F, Piussi P, Remacle J, de Santo A V. Litter mass loss rates in pine forests of Europe and eastern United States: some relationships with climate and litter quality. Biogeochemistry, 1993, 20 ( 3 ): 127- 159 .

[43] Monk C D, Gabrieison F C. Effects of shade, litter and root competition on old-field vegetation in South Carolina. Bulletin of the Torrey Botanical Club, 1985, 112(4) : 383-392.

[44] Freschet G T, Aerts R, Cornelissen J H C. A plant economics spectrum of litter decomposability. Functional Ecology, 2012, 26 (1) : 56-65

[45] Wieder W R, Cleveland C C, Townsend A R. Tropical tree species composition affects the oxidation of dissolved organic matter from litter. Biogeochemistry, 2008, 88(2) : 127-138.

[46] Austin A T, Ballaré C L. Dual role of lignin in plant litter decomposition in terrestrial ecosystems. Proceedings of the National Academy of Sciences of the United States of America, $2010,107(10): 4618-4622$.

[47] Strickland M S, Osburn E, Lauber C, Fierer N, Bradford M A. Litter quality is in the eye of the beholder: initial decomposition rates as a function of inoculum characteristics. Functional Ecology, $2009,23(3): 627-636$.

[48] Liu P, Huang J H, Sun O J, Han X G. Litter decomposition and nutrient release as affected by soil nitrogen availability and litter quality in a semiarid grassland ecosystem. Oecologia, 2010, 162 (3) : 771-780.

[49] Li Y L, Meng Q T, Zhao X Y, Cui J H. Relationships of fresh leaf traits and leaf litter decomposition in Kerqin sandy land. Acta Ecologica Sinica, 2008, 28(6) : 2486-2492.

[50] Huang Y, Shen Y, Zhou M, Ma R S. Decomposition of plant residue as influenced by its lignin and nitrogen. Acta Phytoecologica Sinica, 2003, 27(2) : 183-188.

[51] Zhang D Q, Hui D F, Luo Y Q, Zhou G Y. Rates of litter decomposition in terrestrial ecosystems: global patterns and controlling factors. Journal of Plant Ecology, 2008, 1(2) : 85-93.

[52 ] Li L J, Zeng D H, Yu Z Y, Fan Z P, Yang D, Liu Y X. Impact of litter quality and soil nutrient availability on leaf decomposition rate in a semi-arid grassland of Northeast China. Journal of Arid Environments, 2011, 75(9) : 787-792.

[53] Berg B, Matzner E. Effect of $\mathrm{N}$ deposition on decomposition of plant litter and soil organic matter in forest systems. Environmental Reviews, 1997, 5(1): 1-25.

[54] Liu P, Sun O J, Huang J H, Li L H, Han X G. Nonadditive effects of litter mixtures on decomposition and correlation with initial litter $\mathrm{N}$ and $\mathrm{P}$ concentrations in grassland plant species of northern China. Biology and Fertility of Soils, 2007, 44 (1): 211-216.

[55] Chen H, Harmon M E, Griffiths R P. Effects of temperature and moisture on carton respired from decomposing woody roots. Forest Ecology and Management, 2000, 138(1/3): 51-64.

[56] Berg B, Steffen K T, McClaugherty C. Litter decomposition rate is dependent on litter Mn concentrations. Biogeochemistry, 2007, 82 ( 1 ) : 29-39.

[57] Horner J D, Gosz J R, Cates R G. The role of carbon-based plant secondary metabolites in decomposition in terrestrial ecosystems. The American Naturalist, 1988, 132(6) : 869-883.

[58 ] Hooper D U, Vitousek P M. The effects of plant composition and diversity on ecosystem processes. Science, 1997, 277 (5330): 1302-1305.

[59] Zak D R, Holmes W E, White D C, Peacock A D, Tilman D. Plant Diversity, Soil Microbial Communities, and ecosystem function: are there any links?. Ecology, 2003, 84 ( 8 ): 2042-2050.

[60] Wardle D A, Yeates G W, Barker G M, Bonner K I. The influence of plant litter diversity on decomposer abundance and diversity. Soil Biology and Biochemistry, 2006, 38 ( 5 ): 1052-1062.

[61] Hättenschwiler S, Tiunov A V, Scheu S. Biodiversity and litter decomposition in terrestrial ecosystems. Annual Review of Ecology Evolution System, 36: 191-218.

[62 ] McArthur J V, Aho J M, Rader R B, Mills G L. Interspecific leaf interactions during decomposition in aquatic and floodplain 
ecosystems. Journal of the North American Benthological Society, 1994, 13(1) : 57-67.

[63] Nilsson M C, Wardle D A, Dahlberg A. Effects of plant litter species composition and diversity on the boreal forest plant-soil system. Oikos, 1999, 86(1): 16-26.

[64] Blair J M, Parmelee R W, Beare M H. Decay rates, nitrogen fluxes, and decomposer communities of single- and mixed-species foliar litter. Ecology, 1990, 71(5) : 1976-1985.

[65] Scherer-Lorenzen M, Bonilla J B, Potvin C. Tree species richness affects litter production and decomposition rates in a tropical biodiversity experiment. Oikos, 2007, 116(12) : 2108-2124.

[66] Gessner M O, Swan C M, Dang C K, Mckie B G, Bardgett R D, Wall D H, Hättenschwiler S. Diversity meets decomposition. Trends in Ecology and Evolution, 2010, 25(6) : 372-380.

[67] Wu H T, Lu X G, Yang Q, Jiang M. Ecological characteristics and functions of soil fauna community. Acta Pedologica Sinica, 2006, 43(2): 314-323.

[68] Wu H T, Lu X G, Yang Q, Jiang M, Tong S Z. The early-stage litter decomposition and its influencing factors in the wetland of the Sanjiang Plan, China. Acta Ecologica Sinica, 2007, 27 ( 10$)$ : 4027-4035.

[69] Anderson J M. The breakdown and decomposition of sweet chestnut (Castanea sativa Mill.) and beech (Fagus sylvatica L.) leaf litter in two deciduous woodland soils. Oeeologia, 1973, 12 (3) : 251-274.

[70] Verhoef H A, Brussaard L. Decomposition and nitrogen mineralization in natural and agroecosystem: the contribution of soil animals. Biogeochemistry, 1990, 11(3) : 175-211.

[71] Loranger G, Ponge J F, Imbert D, Lavelle P. Leaf decomposition in two semi-evergreen tropical forests: influence of litter quality. Biology and Fertility of Soils, 2002, 35(4) : 247-252.

[72] Dang C K, Chauvet E, Gessner M O. Magnitude and variability of process rates in fungal diversity-litter decomposition relationships. Ecology Letters, 2005, 8(11) : 1129-1137.

[73] de Boer W, Folman L B, Summerbell R C, Boddy L. Living in a fungal world: impact of fungi on soil bacterial niche development. FEMS Microbiology Reviews, 2005, 29(4) : 795-811.

[74] Osono T. Ecology of ligninolytic fungi associated with leaf litter decomposition. Ecological Research, 2007, 22(6) : 955-974.

[75] Couteaux M M, Bottner B, Berg B. Litter decomposition, climate and litter quality. Trends in Ecology and Evolution, 1995, 10 (2) : 63-66.

[76] Moore T R, Trofymow J A, Taylor B, Prescott C, Camire C, Duschene L, Fyles J, Kozak L, Kranabetter M, Morrison I, Sittanen M, Smith S, Titus B, Visser S, Wein R, Zoltai S. Litter decomposition rates in Canadian forests. Global Change Biology, $1999,5(1): 75-82$.

[77] Edwards A C, Cresser M S. Freezing and its effect on chemical and biological properties of soil. Advances in Soil Sciences, 1992,
18: $59-79$.

[78] Zhang B, Yao S H, Hu F. Microbial biomass dynamics and soil wettability as affected by the intensity and frequency of wetting and drying during straw decomposition. European Journal of Soil Science, 2007, 58(6): 1482-1492.

[79] Hobbie S E. Contrasting effects of substrate and fertilizer nitrogen on the early stages of litter decomposition. Ecosystems, 2005, 8 (6) : 644-656.

[80] Knorr M, Frey S D, Curtis P S. Nitrogen additions and litter decomposition: a meta-analysis. Ecology, 2005, 86 ( 12 ): 3252-3257.

[81] Hobbie S E. Nitrogen effects on decomposition: a five-year experiment in eight temperate sites. Ecology, 2008, 89 (9): 2633-2644.

[82] Manzoni S, Trofymow J A, Jackson R B, Porporato A. Stoichiometric controls on carbon, nitrogen, and phosphorus dynamics in decomposing litter. Ecological Monographs, 2010, 80 (1) : 89-106.

[83] Mckinley V L, Veatal J R. Effects of acid on plant litter decomposition in an arctic lake. Applied and Environmental Microbiology, 1982, 43(5) : 1188-1195.

[84] Santos P F, Elkins N Z, Steinberger Y, Whitford W G. A comparison of surface and buried Larrea tridentata leaf litter decomposition in North American hot deserts. Ecology, 1984, 65 (1) : 278-284.

[85] Zhou D W, Zhou Y L. Fire effects on decomposition and accumulation of litter in Aneurolepidium chinense steppe. Acta Prataculturae Sinica, 1993, 2(4) : 51-55.

[86] Lü X T, Lü F M, Zhou L S, Han X, Han X G. Stoichiometric response of dominant grasses to fire and mowing in a semi-arid grassland. Journal of Arid Environment, 2012, 78: 154-160.

[87] Guo J X, Shi S. Ecohydrological functions of litter in three main plant communities on Songnen grassland. Chinese Journal of Applied Ecology, 2007, 18(8) : 1722-1726.

[88] Facelli J M, Pickett S T A. Plant litter: light interception and effects on an old-field plant community. Ecology, 1991, 72(3): 1024-1031.

[89] Reader R J. Control of seedling emergence by ground cover and seed predation in relation to seed size for some old-field species. Journal of Ecology, 1993, 81(1) : 169-175.

[90 ] Bosy J L, Reader R J. Mechanisms underlying the suppression of forb seedling emergence by grass (Poa pratensis) litter. Functional Ecology, 1995, 9(4) : 635-639.

[91] Deng Y, Jiang Z C, Qin X M, Qi X F, Lan F N, Wu H Y. Impacts of litter on physical and chemical soil properties and its karst effects on different forested Karst ecosrstem. Acta Ecologica Sinica, 2009, 29(6) : 3307-3315.

[92] Kuzyakov Y. Sources of $\mathrm{CO}_{2}$ efflux from soil and review of partitioning methods. Soil Biology and Biochemistry, 2006, 38 
(3) : 425-448.

[93] Villalobos-Vega R, Golgstein G, Haridasan M, Franco A C, Miralles-Wilhelm F, Scholz F G, Bucci S J. Leaf litter manipulations alter soil physicochemical properties and tree growth in a Neotropical savanna. Plant Soil, 2011, 346(1) : 385-397.

[94] Herranz J M, Ferrandis P, Copete M A, Duro E M, Zalacaín A. Effect of allelopathic compounds produced by Cistus ladanifer on germination of 20 Mediterranean taxa. Plant Ecology, 2006, 184 (2) : 259-272

[95] Orwin K H, Buckland S M, Johnson D, Turner B L, Smart S, Oakley S, Bardgett R D. Linkages of plant traits to soil properties and the functioning of temperate grassland. Journal of Ecology, 2010, 98(5) : 1074- 1083 .

[96] Loydi A, Eckstein R L, Otte A, Donath T W. Effects of litter on seedling establishment in natural and semi-Natural grassland: a meta-analysis. Journal of Ecology, 2012, 101(2) : 454-464.

[97] Eckstein R L, Donath T W. Interactions between litter and water availability affect seedling emergence in four familial pairs of floodplain species. Journal of Ecology, 2005, 93(4) : 807-816.

[98] Jensen K, Gutekunst K. Effects of litter on establishment of grassland plant species: The role of seed size and successional status. Basic and Applied Ecology, 2003, 4(6) : 579-587.

\section{参考文献:}

[ 9 ] 占峰, 杨冬梅. 光照条件、植株冠层结构和枝条寿命的关系一 以桂花和水杉为例. 生态学报, 2012, 32(3) : 984-992.

[26] 李博. 中国北方草地退化及其防治对策. 中国农业科学, 1997, 30(6) : 1-9.

[27] 刘国华, 傅伯杰, 陈利顶, 郭旭东. 中国生态退化的主要类 型、特征及分布. 生态学报, 2000, 20(1)：13-19.

[49］李玉霖, 孟庆涛, 赵学勇, 崔建垣. 科尔沁沙地植物成熟叶片 性状与叶调落物分解的关系. 生态学报, 2008, 28 (6): 2486-2492

[50] 黄耀, 沈雨, 周密, 马瑞升. 木质素和氮含量对植物残体分解 的影响. 植物生态学报, 2003, 27(2)：183-188.

[67] 武海涛, 吕宪国, 杨青, 姜明. 土壤动物主要生态特征与生态 功能研究进展. 土壤学报, 2006, 43(2) : 314-323.

［68］武海涛，吕宪国，杨青，姜明，佟守正.三江平原典型湿地枯 落物早期分解过程及影响因素. 生态学报, 2007, 27(10): 4027-4035.

[85] 周道玮，周以良. 羊草草甸草原火烧地调落物的分解与积累 速率变化. 草业学报, 1993, 2(4) : 51-55.

[87] 郭继勋, 施爽. 松嫩草原三种主要植物群落枯落物层生态水 文功能. 应用生态学报, 2007, 18(8)：1722-1726.

[91] 邓艳, 蒋忠诚, 覃星铭, 祁晓凡, 蓝芙宁, 吴华英. 岩溶生态 系统中不同植被枯落物对土壤理化性质的影响及岩溶效应. 生态学报, 2009, 29(6): 3307-3315. 